\title{
Dickschicht Sulfidsensor auf Basis einer Chalkogenidglas-ISE
}

\author{
U. Enseleit ${ }^{1}$, C. Feller ${ }^{2}$, U. Partsch ${ }^{2}, \underline{\text { W. Vonau }}{ }^{1}$ \\ ${ }^{1}$ Kurt-Schwabe-Institut für Mess- und Sensortechnik Meinsberg e.V., Waldheim, Waldheim, Deutschland \\ ${ }^{2}$ Fraunhofer Institut für Keramische Technologien und Systeme, Dresden, Germany, Deutschland \\ Kontakt: ute.enseleit@ksi-meinsberg.de
}

\section{Einleitung}

Die quantitative Sulfidionenbestimmung ist von großer praktischer Bedeutung. So spielt sie z. B. bei der LangzeitIn-Situ-Überwachung von Thermalbädern, geothermalen Böhrlöchern und Abwassernetzen eine nicht unbedeutende Rolle. Für die Bestimmung von Sulfid in Lösung werden $u$. a. sowohl kolorimetrische und iodometrische Methoden als auch ein potentiometrisches Verfahren unter Verwendung einer konventionellen Silbersulfid-Elektrode nach Probenhandhabung und Konditionierung auf hohen $\mathrm{pH}-$ Wert beschrieben [1]. Ebenso wurde schon über die Verfügbarkeit anionenselektiver Chalkogenidglas (CG)-Elektroden zur Bestimmung von Gesamtsulfid-, Bromid- und lodidionen in wässrigen Lösung berichtet [2-5]. Bei diesen Sensoren handelt es sich um stabförmige CG-Sensoren, wobei geschmolzene CG-Stücke mechanisch zerkleinert, elektrisch kontaktiert, armiert und ggf. plan geschliffen werden. Dieses Herstellungsverfahren ist kostenintensiv und zeitaufwendig. Das Ziel unserer Arbeit ist es, eine geeignete Glaskomposition aufzufinden und technologische Prozesse zu entwickeln, um zu in Dickschicht (DS)-Technik herstellbaren planaren, sulfidionenselektiven CG-Elektroden zu gelangen. Vorteile eines Sulfid-DS-Sensors beruhen auf seiner mechanischen Robustheit und hohen chemischen Beständigkeit, auch ist perspektivisch die Möglichkeit der Integration mehrerer Sensormembranen auf Basis von CGs, sowie z. B. eines pH-sensitiven Glases und eines Temperatursensors auf einem Chip zum simultanen Nachweis von Anionen oder Kationen, des $\mathrm{pH}$-Wertes und der Temperatur gegeben $[6,7]$.

\section{Experimentelles}

Bei der Bestimmung von Sulfiden in wässrigen Lösungen sind das chemische Gleichgewicht zwischen verschiedenen Sulfidspezies in Abhängigkeit vom pH-Wert und auftretende Redoxprozesse in Gegenwart von Sauerstoff zu beachten. Die zweistufige Dissoziation des gelösten Schwefelwasserstoffs wird durch die Gleichungen 1 und 2 und die Gleichgewichtskonstanten $\left(\mathrm{K}_{1}\right.$ und $\left.\mathrm{K}_{2}\right)$ beschrieben:

$$
\begin{array}{ll}
\mathrm{H}_{2} \mathrm{~S}+\mathrm{H}_{2} \mathrm{O} \leftrightarrow \mathrm{H}_{3} \mathrm{O}^{+}+\mathrm{HS}^{-} & K_{1}=1,1 \times 10^{-7} \\
\mathrm{HS}^{-}+\mathrm{H}_{2} \mathrm{O} \leftrightarrow \mathrm{H}_{3} \mathrm{O}^{+}+\mathrm{S}^{2-} & K_{2}=1,3 \times 10^{-13}
\end{array}
$$

Unterhalb von pH 5 liegt hauptsächlich das gelöste Gas $\mathrm{H}_{2} \mathrm{~S}$ vor bzw. wird es freigesetzt. Die dominierende Spezies bei $7<\mathrm{pH}<13$ ist $\mathrm{HS}^{-}$, während $\mathrm{S}^{2-}$-Ionen nur oberhalb von $\mathrm{pH} 12$ in einem signifikanten Anteil auftreten. Alle Sulfidspezies werden im oxidierenden Medium oder bei Anwesenheit von Sauerstoff zu elementaren Schwefel und weiter zu $\mathrm{SO}_{3}{ }^{2-}$ bzw. $\mathrm{zu} \mathrm{SO}_{4}{ }^{2-}$ oxidiert. Die Gesamtsulfidkonzentration $\mathrm{S}_{\mathrm{T}}$ in Lösung kann somit wie folgt ausgedrückt werden:

$\mathrm{S}_{\mathrm{T}}=\left[\mathrm{S}^{2-}\right]+\left[\mathrm{HS}^{-}\right]+\left[\mathrm{H}_{2} \mathrm{~S}\right]$

Durch die Entwicklung der selektiven DS-Elektroden auf CG-Basis soll vor allem das im neutralen $\mathrm{pH}$-Bereich existierende $\mathrm{HS}^{-}$, z. B. in Abwasserreinigungsanlagen, in natürlichen Gewässern oder im Meerwasser potentiometrisch detektiert bzw. online überwacht werden können.

\section{Material und Methoden}

Für die Herstellung der CGs wurden CdS-, Agl-, $\mathrm{Ag}_{2} \mathrm{~S}-$ und $\mathrm{As}_{2} \mathrm{~S}_{3}$-Pulver verwendet, jeweils in einer Reinheit von > 99.99 Gew.-\%. Das Schmelzen des Sensormaterials der Zusammensetzung $4 \mathrm{CdS}$ - $24 \mathrm{Agl}-25 \mathrm{Ag}_{2} \mathrm{~S}-47 \mathrm{As}_{2} \mathrm{~S}_{3}$ (mol \%) erfolgte in einer zylindrischen Quarzampulle. Diese wurde in eine Reaktionskammer mit Stickstoff gefüllt, evakuiert $\left(<10^{-3}\right.$ mbar) und hermetisch abgedichtet. Das Pulvergemenge wurde in einen Quarzglasreaktor überführt, der dann in einen Rohrofen eingebracht wurde. Für die langsame Bewegung der Ampulle während der Aufheizphase mit 100 bis $110^{\circ} \mathrm{C} / \mathrm{h}$ bis zu einer Temperatur von $980^{\circ} \mathrm{C}$ und einer Schmelzprozessdauer von $20 \mathrm{~h}$ wurde ein Rührer verwendet. Unmittelbar nach dem Schmelzvorgang wurde die Ampulle in kaltem Wasser abgeschreckt und zur Sammlung des Glases zerstört.

Mit Methoden der Thermoanalytik wurde geprüft, ob das CG mit Dickschichttechnologie prozessierbar und zu einer dichtsinternden Glasmembran verarbeitbar ist. Das thermische Zersetzungsverhalten des CGs wurde mittels simultaner Thermogravimetrie-Differenzthermoanalyse mit dem Gerät STA 449 C der Firma NETZSCH-Gerätebau GmbH im Temperaturbereich von $15^{\circ} \mathrm{C}$ bis $820^{\circ} \mathrm{C}$ unter Stickstoffatmosphäre am CG-Pulver untersucht.

Mit der Thermomechanischen Analyse TMA 402 F1 Hyperion, ebenfalls von NETZSCH-Gerätebau $\mathrm{GmbH}$, wurde das Sinterverhalten des CG-Glaspulvers unter Stickstoff bestimmt. Dafür sind zylindrische Pulverpresslinge mittels uniaxialen Pressens hergestellt und vermessen worden.

Mit Hilfe der Röntgendiffraktometrie (XRD) mit dem Gerät D8 advance der Firma Bruker wurde überprüft, ob die CG im amorphen oder kristallinen Zustand vorliegen.

Die Abscheidung einer CG-Membran in DS-Technik auf einem Keramiksubstrat (Abb. 1) erfordert die Verarbeitung einer Glaspaste, wofür Glaspulver benötigt wird. Dazu wurde das CG-Bulkmaterial in einer Planetenkugelmühle trocken zum Glaspulver gemahlen. Für die Herstellung der 
Glaspaste ist ein organisches Vehikel verwendet worden. Dieses wurde durch Einrühren des Polymers Methacrylatharz in das Lösungsmittel Terpineol bei $80^{\circ} \mathrm{C}$ hergestellt.

Die Pastenherstellung erfolgte durch Dispergieren des Glaspulvers in dem organischen Vehikel. Um das Pulver fein zu dispergieren und zu deagglomerieren, wurde die Paste fünfmal auf einem Dreiwalzwerk 50EC (EXAKT Advanced Technologies) bearbeitet. Das Mahlgut ist bis zu einer Korngröße $<30 \mu \mathrm{m}$ dispergiert worden. Die Glaspaste wurde im Siebdruckverfahren auf ein $\mathrm{ZrO}_{2}$-Substrat über eine Goldschicht gedruckt. Der Einbrand der CG-Schicht erfolgte entsprechend der Sintertemperatur des Glases.
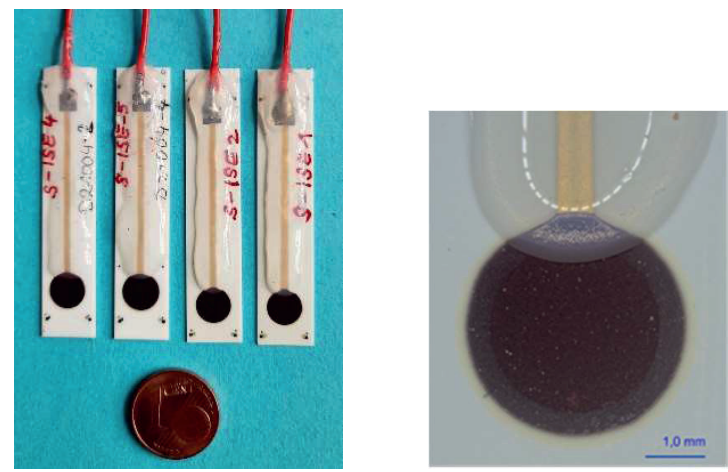

Abb. 1: Prototypen von DS-Elektroden (links), sensitive Elektrodenoberfläche (rechts)

Zur Verhinderung einer Sulfidoxidation bei den potentiometrischen Messungen wurde bei der Herstellung der Kalibrierlösungen unter Verwendung von $\mathrm{Na}_{2} \mathrm{~S} \times 9 \mathrm{H}_{2} \mathrm{O}$ mit einer Reinheit von 98 Masse-\%, gekochtes und entgastes deionisiertes Wasser verwendet, um den gelösten Sauerstoff und $\mathrm{CO}_{2} \mathrm{zu}$ entfernen. Die Lösungen wurden jeden Tag frisch hergestellt. Innerhalb von 1 bis 2 Stunden wurden unter Luftabschluss die potentiometrischen Messungen in einer Zelle vorgenommen. Die pH-Werte der SulfidKalibrierlösungen wurden mit $10^{-1} \mathrm{M} \mathrm{NaOH}, 10^{-1} \mathrm{M} \mathrm{HNO}_{3}$ und mit TRIS-Puffer eingestellt und mit jeweils $10^{-1} \mathrm{M} \mathrm{KNO}_{3}$ zur Einstellung der lonenstärke versehen. Es wurde mit einer auf $10^{-1} \mathrm{M} \mathrm{KNO}_{3}$ basierenden Salzbrücke gearbeitet, um die Referenzelektrode von der Messlösung zu trennen und das Ausfällen, z. B. von Silberchlorid, zu verhindern.

Für die Untersuchung des elektrochemischen Verhaltens des Sensors gegenüber $\mathrm{Cd}^{2+}$-lonen wurden aus einer $10^{-1}$ $\mathrm{M} \mathrm{Cd}\left(\mathrm{NO}_{3}\right)_{2}$-Stammlösung Kalibrierlösungen im Messbereich von $10^{-2} \mathrm{M}_{\text {bis }} 10^{-6} \mathrm{M} \mathrm{Cd}^{2+}$ mit jeweils $10^{-1} \mathrm{M} \mathrm{KNO}_{3}$ zur Einstellung der lonenstärke nach der Verdünnungsmethode hergestellt. Als Messgerät zur Elektrodenpotentialmessung und zur Datenaufzeichnung diente das Messsystem LM 3000 von der Sensortechnik Meinsberg $\mathrm{GmbH}$ (Xylem Analytics Germany Sales $\mathrm{GmbH} \&$ Co. KG).

Die Querempfindlichkeit des DS-Sensors gegenüber den Störionen $\mathrm{KCl}, \mathrm{KHCO}_{3}, \mathrm{KBr}$ und $\mathrm{KI}$ wurde nach der von der IUPAC empfohlenen Methode mit gemischten Lösungen bestimmt und die Selektivitätskoeffizienten wurden ermittelt.

\section{Resultate und Diskussion}

Abb. 2 zeigt ein Röntgenbeugungsdiagramm von einer Pulverprobe des CG. Die leicht geschwungene Reflexionslinie weist keine sichtbaren Peaks bzw. Reflexe auf. Hiermit wird ein amorpher Charakter verbunden mit einer hohen Stabilität des Glases in wässrigen Lösungen bestätigt.

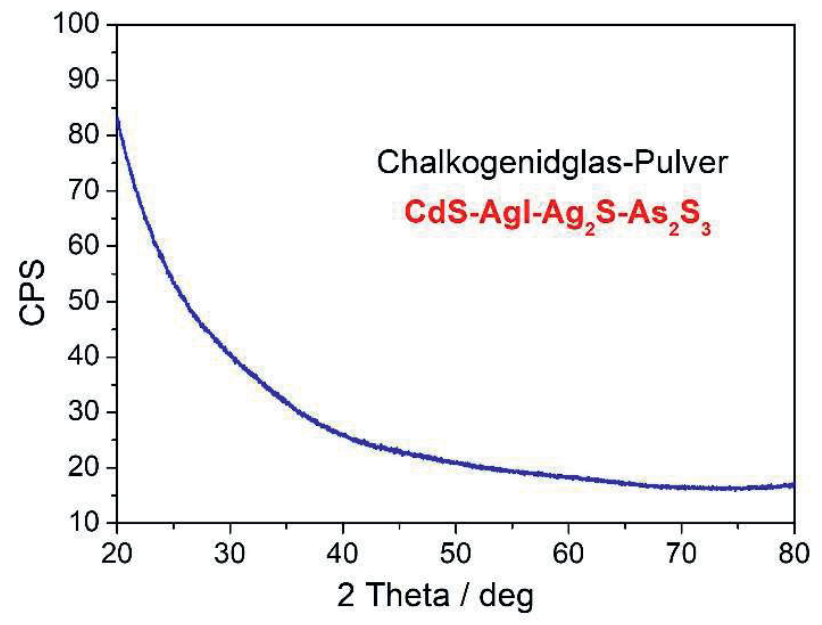

Abb. 2: Rötgenbeugungsdiagramm von Sulfid-sensitiven CG-Pulver

Die thermogravimetrische Untersuchung zeigt, dass das CG unter Stickstoff bis $320{ }^{\circ} \mathrm{C}$ thermisch stabil ist. Bei höheren Temperaturen tritt eine deutliche Masseabnahme auf, was auf die thermische Instabilität und Zersetzung des Glases hinweist (Abb. 3). Die mittels simultan durchgeführter Differenzthermoanalyse erhaltene DTA-Kurve zeigt bei $230{ }^{\circ} \mathrm{C}$ ein schwaches und bei $270{ }^{\circ} \mathrm{C}$ ein starkes exothermes Signal, wodurch eine neue Phasenbildung oder Kristallisation angezeigt wird. Bei $350{ }^{\circ} \mathrm{C}$ weist wiederum ein starkes endothermes Signal auf eine Phasenumwandlung oder Zersetzung hin.

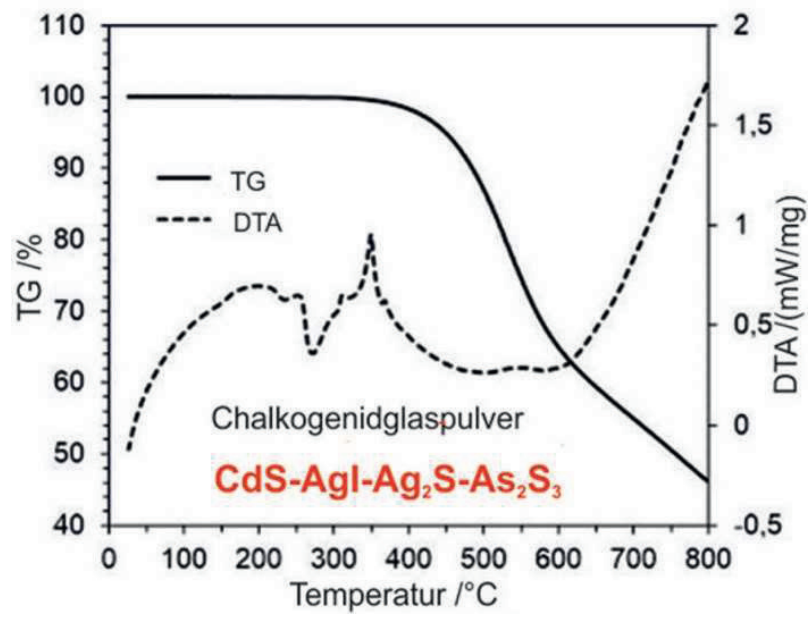

Abb. 3: Thermisches- und Sinterverhalten von Sulfidsensitiven CG-Pulver

Für den Dickschichtprozess ist nur thermisch stabiles Glasmaterial geeignet, das während des Sinterns unter Beibehaltung der Stöchiometrie verdichtet werden kann. Für CdS-Agl-Ag 2 S- $\mathrm{As}_{2} \mathrm{~S}_{3}-\mathrm{CG}$ trifft dies zu. Das Glas kann bei 
$185^{\circ} \mathrm{C}$ zu einer dichten Glasmembran versintert werden. Thermische Effekte durch Zersetzung, Kristallisation und Phasenumwandlung des CGs treten erst oberhalb der Sintertemperatur auf.

Das Ansprechverhalten einer neuen CG-DS-ISE in Natriumsulfid Kalibrierlösungen, die mit TRIS Puffer auf einen neutralen $\mathrm{pH}$-Wert $(7,3)$ hergestellt wurden, ist in Abb. 4 dargestellt. Die Steigung der DS-ISE Messkette im linearen Messereich von $10^{-1} \mathrm{M}$ bis ca. $10^{-5} \mathrm{M} \mathrm{Na}_{2} \mathrm{~S} \times 9 \mathrm{H}_{2} \mathrm{O}, 10^{-1} \mathrm{M}$ $\mathrm{KNO}_{3}$ bei neutralem $\mathrm{pH}$-Wert mit $60 \pm 0,5 \mathrm{mV} / \mathrm{pS}$ T stimmt gut mit der Nernst-Gleichung für einwertige Ionen $\left(\mathrm{HS}^{-}\right)$ überein.

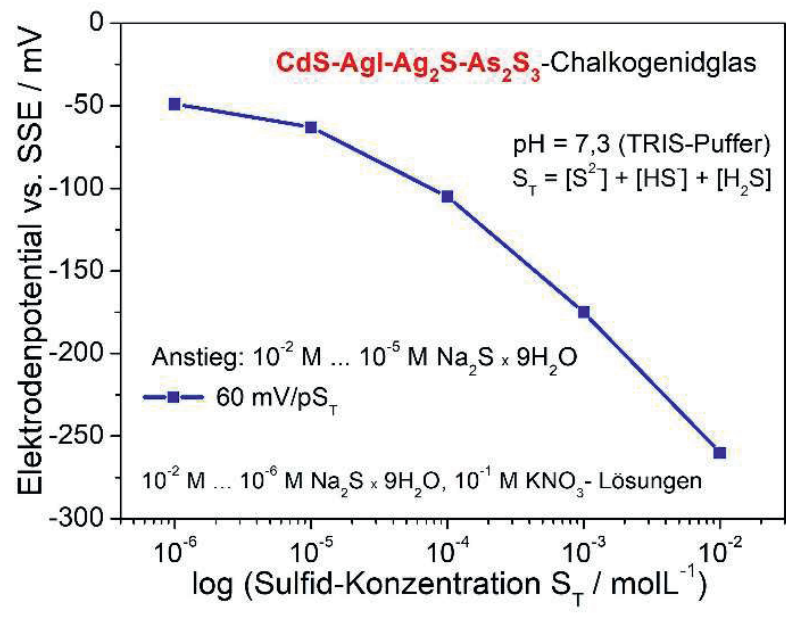

Abb. 4: Kalibrierkurve einer CG-Sulfid-ISE bei einem $\mathrm{pH}-$ Wert von $\mathrm{pH} \mathrm{7,3}$

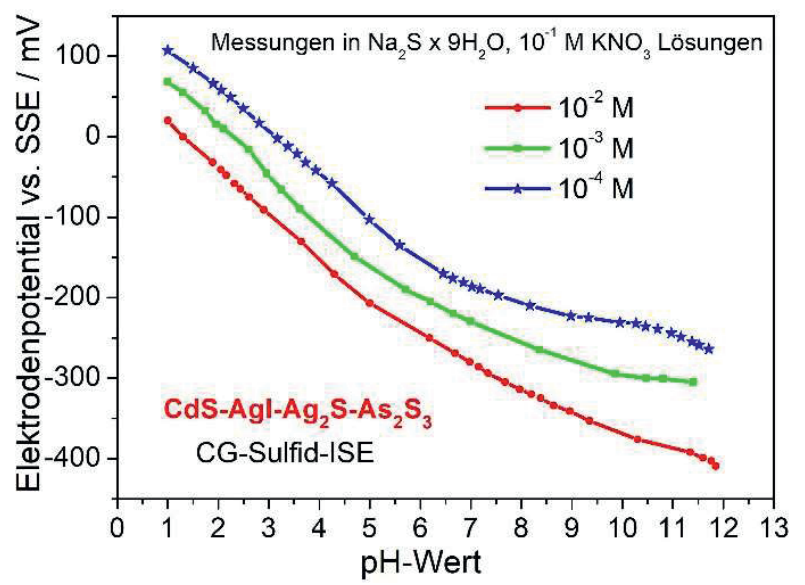

Abb. 5: pH-Abhängigkeit des Messsignals einer CGSulfid-ISE

Weiterhin wurden Messungen in Lösungen unterschiedlicher Konzentrationen an Gesamtsulfid von $10^{-4} \mathrm{M}, 10^{-3} \mathrm{M}$, und $10^{-2} \mathrm{M}$ bei verschiedenen $\mathrm{pH}-$ Werten durchgeführt. Abb. 5 zeigt einen konstanten Spannungsabfall von ca. 60 $\mathrm{mV}$ zwischen den drei Kurven mit dem Ergebnis, dass der Sensor im gesamten untersuchten $\mathrm{pH}$-Bereich empfindlich ist.

Außerdem kann man im pH-Bereich von 1 bis 7 einen starken Abfall des Potentials beobachten, während für $\mathrm{pH}$
$>7$ nur eine geringfügige Potentialänderung stattfindet. Entsprechend dem Gleichgewicht zwischen den verschiedenen Sulfid-Spezies steigt bei einem gegebenen $\mathrm{S}_{\mathrm{T}}$ die HS--Konzentration linear über den pH-Bereich von 3 bis 7 an, um schließlich einen nahezu konstanten Wert über $\mathrm{pH}$ $7 \mathrm{zu}$ erreichen. Dies ist ein Hinweis, dass die Sulfid-ISE empfindlich gegenüber HS-Spezies ist.

Weiterhin wurde eine Empfindlichkeit des CdS-Agl-Ag2 S$\mathrm{As}_{2} \mathrm{~S}_{3}-\mathrm{CG}$ gegenüber Cadmiumionen festgestellt. Die Kalibrierkurve einer entsprechenden DS-Elektrode ist in Abb. 6 dargestellt. Es wurde eine Elektrodenempfindlichkeit von $26 \mathrm{mV} / \mathrm{pCd}^{2+}$ in Kalibrierlösungen von $10^{-2} \mathrm{M}$ bis $10^{-6} \mathrm{M}$ $\mathrm{Cd}\left(\mathrm{NO}_{3}\right)_{2}, 10^{-1} \mathrm{M} \mathrm{KNO}_{3}$ bestimmt. Dieser Umstand muss für praktische Messungen in Betracht gezogen werden.

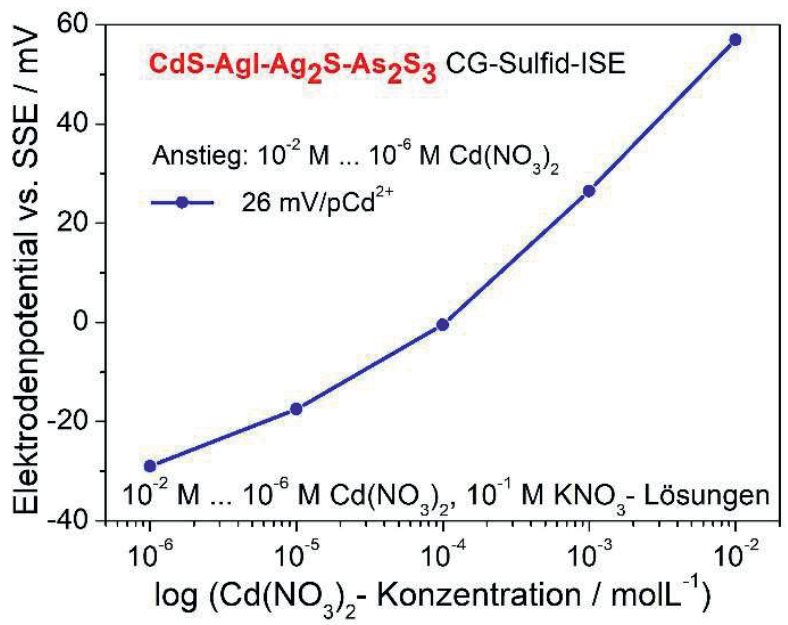

Abb. 6: Querempfindlichkeit gegenüber Cadmiumionen

Die Selektivitätskoeffizienten eines DS-Sensors auf Basis des Materialsystems CdS-Agl- $\mathrm{Ag}_{2} \mathrm{~S}-\mathrm{As}_{2} \mathrm{~S}_{3}$ in einem Messbereich von $10^{-2} \mathrm{M}$ bis $10^{-6} \mathrm{M} \mathrm{Na} 2 \mathrm{~S} \times 9 \mathrm{H}_{2} \mathrm{O}, 10^{-1} \mathrm{M} \mathrm{KNO}_{3}$ unter Zusatz einer konstanten Konzentration von $10^{-2} \mathrm{M}$ an Störionen $\left(\mathrm{KCl}, \mathrm{KHCO}_{3}, \mathrm{KBr}, \mathrm{KI}\right)$ sind aus Tabelle 1 ersichtlich.

Tabelle 1: Selektivitätskoeffizienten einer Sulfid-GC-ISE gegen verschiedene Störionen (S)

\begin{tabular}{|c|c|}
\hline Störion S & Selektivitätskoeffizient \\
\hline $\mathrm{Cl}^{-}$ & $2 \times 10^{-5}$ \\
\hline $\mathrm{Br}^{-}$ & $2 \times 10^{-3}$ \\
\hline $\mathrm{I}^{-}$ & $1 \times 10^{-4}$ \\
\hline $\mathrm{HCO}_{3}^{-}$ & $3 \times 10^{-3}$ \\
\hline
\end{tabular}

Die Kurvenverläufe in Abb. 7 zeigen, dass bei niedrigen Sulfidionen-Konzentrationen ab ca. $10^{-4} \mathrm{M}$, die Anwesenheit von Störionen in einer hohen Konzentration $\left(10^{-2} \mathrm{M}\right)$, das Elektrodenpotential der Elektrode signifikant beeinflussen können. 


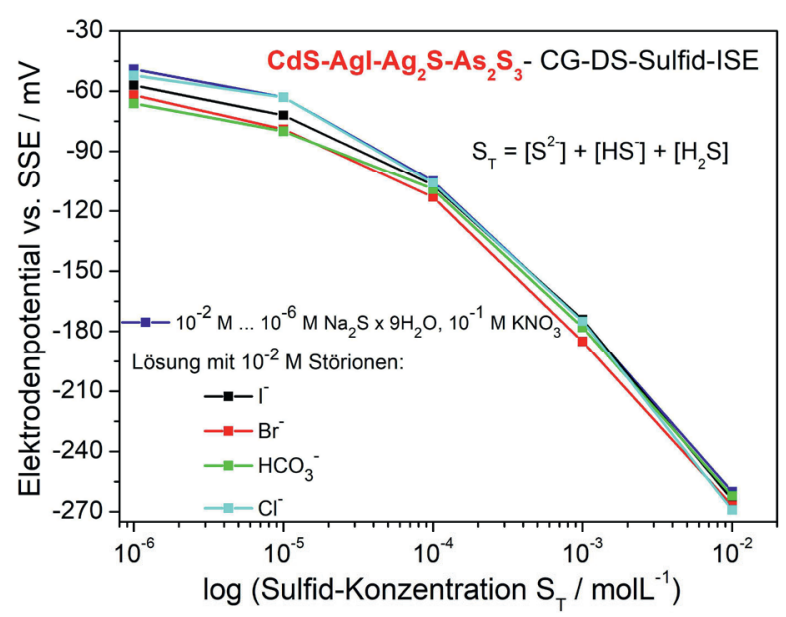

Abb. 7: Bestimmung der Selektivitätskoeffizienten nach der Methode mit gemischten Lösungen

\section{Zusammenfassung}

Die Entwicklung und Verifizierung eines potentiometrischen Dickschichtsensors auf Chalkogenidglas-Basis mit einer neuartigen Zusammensetzung zur Bestimmung von Sulfidionen in wässrigen Medien wurde vorgestellt. Es handelte sich um Materialien auf der Grundlage des Stoffsystems CdS-Agl-Ag 2 S-As ${ }_{2} S_{3}$, die für die Verarbeitung zu einer Glaspaste und deren Siebdruck und Einbrand auf ein Keramiksubstrat zur Herstellung einer dichten Glasmembran geeignet sind. Im Gegensatz zu konventionellen, basierend auf $\mathrm{Ag}_{2} \mathrm{~S}$-haltigen Salzpresslingen hergestellten und für Sulfidionen empfindlichen potentiometrischen Elektroden, wurden für die CG-basierten ISE im neutralen pH-Bereich in einem Konzentrationsbereich von $10^{-2} \mathrm{M}$ bis $10^{-5} \mathrm{M} \mathrm{Na}_{2} \mathrm{~S}$ x $9 \mathrm{H}_{2} \mathrm{O}$ bei Raumtemperatur Elektrodenfunktionen von 60 $\mathrm{mV} / \mathrm{pS}$ T ermittelt. Dies entspricht einer leichten Übersteilheit in Bezug zu dem nach der Nernstschen Gleichung zu erwartenden Wert für die Detektion des dort existierenden HS--lons. Die Sensoren sind somit für eine Prozesskontrolle von zahlreichen Abwässern für direkte Messungen des gelösten Schwefelwasserstoffs geeignet. Außerdem zeichnen sie sich durch hohe mechanische Robustheit und chemische Beständigkeit aus.

Die Möglichkeit, perspektivisch weitere CGs und ein $\mathrm{pH}$ sensitives Glas sowie einen Temperatursensor auf den keramischen Träger mit Hilfe der DS-Technologie aufzubringen, würde die Anwendungsbreite des Sensors zusätzlich erweitern.

\section{Literatur}

[1] POULY, F.; TOURAND, E.: BUISSON, J.-F.; THOMAS, $O$.: An alternative method for the measurement of mineral sulphide in wastewater. In: Talanta 50, (1999), Nr. 4, S. 737-742, doi: 10.1016/S0039-9140(99)00201-5

[2] ESSI, M. M.: Chemical Sensors for the determination of toxic species in solution. In: Chalcogenide Letters 8, (2011), Nr. 1, S. 25-31

[3] ENSELEIT, U.; FELLER, C.; PARTSCH, U.; VONAU, W.: lodide determination with chalcogenide glass electrodes. In: J. of Solid State Electrochem. 25, (2021), Nr. 8, S. 2293-2300, doi: 10.1007/s10008-021-05000-1

[4] MILOSHOVA, M.S.; BALTES, D.; BYCHKOV, E.A.: New chalcogenide glass chemical sensors for $\mathrm{S}^{2-}$ and dissolved $\mathrm{H}_{2} \mathrm{~S}$ monitoring. In: Water Science and Technology 47, (2003), Nr. 2, S. 135-140, doi: 10.2166/wst.2003.0104

[5] CALI, C.; TAILLADES, G.; PRADEL, A.; RIBES, M.: Determination of sulfur species using a glassy-crystalline chalcogenide membrane. In: Sensors and Actuators B76, (2001), S. 560-564, doi: 10.1016/S09254005(01)00631-1

[6] FELLER, C.; PARTSCH, U.: Ceramic multilayer technology as a platform for miniaturized sensor arrays for water analysis. In: J. Sens. Sens. Syst. 10, (2021), S. 8391, doi 10.5194/jsss-10-83-2021

[7] ENSELEIT, U.; BERTHOLD, M.; FELLER, C.; PARTSCH, U.; KÖRNER, S.; VONAU, W.: Chalcogenide Glass Based Heavy Metal Sensors In: Sensors \& Transducers 219, (2018), Nr. 1, S. 1-8, http://www.sensorsportal.com

\section{Danksagung}

Die Arbeiten wurden gefördert vom Bundesministerium für Wirtschaft und Energie (BMWi) über die Arbeitsgemeinschaft industrieller Forschungsvereinigung „Otto von Guericke“ e.V. (AiF) im Rahmen des Programms „Zentrales Innovationsprogramm Mittelstand“ (ZIM)-kooperationen unter dem Förderkennzeichen ZF 4088707AW9.

Das Kurt-Schwabe-Institut für Mess- und Sensortechnik Meinsberg e.V. wird mitfinanziert durch Steuermittel auf der Grundlage des vom Sächsischen Landtag beschlossenen Haushaltes. 